\title{
Key Characteristics of Circulating Tumor Cell Clusters and Implications for Cancer Metastases
}

\author{
Yakov Perlov ${ }^{1}$ and Dean Lee ${ }^{2}$ \\ ${ }^{1}$ Belmont High School. Belmont, MA, USA \\ ${ }^{2} \mathrm{HiFiBiO}$ Therapeutics. Cambridge, MA, USA \\ DOI: https://doi.org/10.47611/jsrhs.v10i2.1470
}

\section{ABSTRACT}

Primary tumors generate metastases by shedding tumor cells into the circulation; these circulating tumor cells (CTCs) implant at distant sites to develop into metastatic lesions. CTCs can travel either as clusters or as single CTCs. Previous studies revealed that the frequency of CTC clusters in a cancer patient positively correlates with the likelihood of developing metastatic lesions. Three key characteristics of CTC clusters - chemoresistance, reduced apoptosis, and epigenetically programmed stemness - enhance their metastatic potential relative to single CTCs:

1. CTC clusters seem to be more resistant to chemotherapy due to their quiescent and necrotic cores, making drug penetration difficult. Their chemoresistance also correlates with specific molecular components of the extracellular matrix.

2. CTC clusters suffer lower rates of apoptosis. This might be attributed to autocrine factors that protect against immune attack and the epithelial-mesenchymal transition.

3. The DNA methylation landscape of CTC clusters closely resembles that of embryonic stem cells. It features hypomethylation of four critical transcription factors associated with stemness and hypermethylation of a set of pro-differentiation genes.

Further research might focus on the interdependence of these three characteristics and whether they precede or follow the clustering of CTCs. The answers to these research questions will help drug developers define specific mechanisms that can curb the metastatic potential of CTC clusters.

\section{Introduction}

Cancer is caused by cells that divide uncontrollably, forming a primary tumor at a single location in the body. Sometimes, these tumors can intravasate into the circulatory system - blood and lymph vessels - and begin shedding cells into the circulation. A small fraction of these circulating tumor cells (CTCs) could travel through the circulation and spawn metastatic lesions in distant sites. (Figure 1) The resulting metastatic tumors, rather than the primary tumor, are often the deadliest features of cancer.

As CTCs are often the source of metastatic tumors, it is essential to understand their biological properties to inform the development of drugs against them. Recent studies have begun to highlight the differences between single CTCs and clusters of CTCs. Initial evidence suggested that CTC clusters have greater metastatic potential than individual CTCs and have distinct biological features that make them so. This review aims to describe the most important features of CTC clusters that enable them to be effective initiators of metastatic tumors.

Chemotherapy is the systemic application of potent chemicals to kill cancer cells. It typically works by damaging the DNA of rapidly dividing cancer cells or blocking essential nutrients from being supplied to cancer cells. Chemotherapy drug classes include alkylating agents, antimetabolites, antitumor antibiotics, topoisomerase inhibitors, and mitotic inhibitors. When cancer cells evade or tolerate these drugs, they are said to have developed 
chemoresistance. One key characteristic of CTC clusters is their remarkable chemoresistance, which makes them more deadly than single CTCs.

Apoptosis is one of the body's most direct defenses against cancer cells. Often described as programmed cell death, apoptosis is the sum of molecular events that lead to the controlled, orderly death of a cell. Both extrinsic and intrinsic factors can induce apoptosis in cancer cells, keeping them at bay. A cell undergoing apoptosis will experience shrinking, nuclear envelope fragmentation, DNA condensation and fragmentation, and RNA decay. While single CTCs do experience apoptosis, CTC clusters have been observed to evade apoptosis consistently. This enhanced survival gives CTC clusters more opportunities to spread throughout the body.

The field of epigenetics includes the study of DNA methylation and a range of histone modifications. These molecular changes regulate gene expression, affecting which genes are expressed and which genes are silenced. By activating and silencing select genes, epigenetic modifications can induce stem cell-like characteristics, or stemness, in CTCs. These characteristics enable the self-renewal of CTCs before they migrate to new sites; they also enable CTCs to differentiate into distinct progenitor cells once they migrate to a new site. CTC clusters exhibit greater stemness than single CTCs; they self-renew to a greater extent and can differentiate upon colonizing a foreign tissue. These properties enable CTC clusters to be more effective transplants to new sites in the body.

These three key characteristics of CTC clusters - chemoresistance, reduced apoptosis, and stemness - make them a topic of fervent interest for many cancer biologists. This review presents these characteristics and discusses their implications for cancer metastasis, as well as possible directions for future research.

\section{Chemoresistance}

To understand the chemoresistance of CTC clusters, Klameth et al. (2017) sought to study them in a laboratory setting. They extracted CTCs specifically from small cell lung cancer (SCLC), a highly metastatic and chemoresistant tumor with unusually high numbers of CTCs. From these SCLC CTCs, they established five cell lines (BHGc7, BHGc10, BHGc16, BHGc26, and UHGc5). CTCs from each patient could grow into large tumorospheres in vitro, which is akin to CTC clusters in vivo. The tumorospheres were immunostained for two proteins - Ki67, a proliferation marker, and CAIX, which possesses a hypoxia-responsive element in its promoter. The immunostaining results showed that cells inside tumorospheres were quiescent (Ki67-low) and hypoxic (CAIX-high), both of which prevent molecules from the environment from reaching the core of the tumorospheres. These findings led Klameth et al. to hypothesize that the tumorospheres are less responsive to chemotherapy drugs than single CTCs were since drug molecules would have difficulty penetrating the quiescent and hypoxic core of the tumorospheres. 


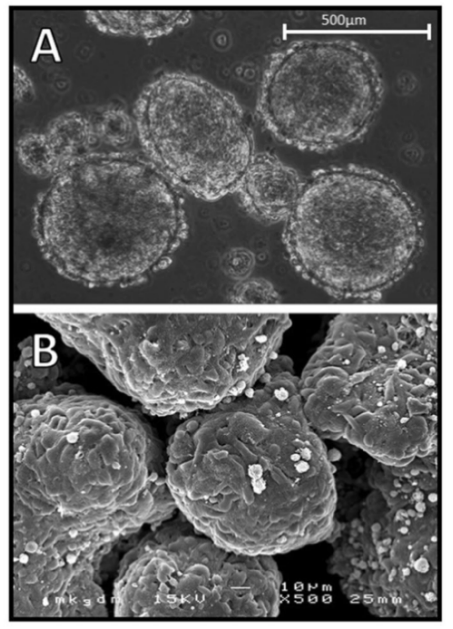

Figure 1. Light and scanning electron microscopy of CTC tumorospheres (A and B) show light and SEM images of BHGc26 tumorospheres, respectively. (Figure from Pampaloni et al., 2007)

A study by Laurent et al. (2013) confirmed that in tumorospheres (which they call spheroids) formed by cells from a pancreatic adenocarcinoma cell line, the tumorosphere cores were also less proliferative and more hypoxic. The authors further connected these two properties of tumorosphere cores to limited cell cycle progression. This suggests that the chemoresistance of CTC clusters is associated with the expression of cyclins, proteins regulating cell cycle progression. Whether decreased cyclin expression sets the stage for the development of chemoresistance remains to be determined.

When Klameth et al. exposed their five CTC cell lines to epirubicin and topotecan, they discovered a significant decrease in tumorospheres' chemosensitivity compared to single-cell suspensions of CTCs. The authors repeated these cytotoxicity tests with two more drugs - cisplatin and etoposide - and found similar decreases in chemosensitivity in the tumorospheres. Comparatively, this study more convincingly demonstrates the chemosensitivity of CTC clusters in comparison to individual CTCs.

Tannock et al. (2002) and Däster et al. (2016) showed other potential contributors to CTC cluster chemoresistance. In the former study, human bladder cancer cells were grown as multicellular layers and treated with five different radio-labeled antitumor drugs: cisplatin, etoposide, gemcitabine, paclitaxel, and vinblastine. There was limited penetration of radio-labeled drug molecules through the multicellular layers compared to control, indicating that extracellular matrix proteins essential for forming multicellular layers might play a role in chemoresistance. Three tumorosphere stages were identified in the latter study, corresponding to different sizes and characterized by normoxia (normal oxygen levels), hypoxia, and hypoxia-induced necrosis. Necrosis is a type of cell death occurring due to uncontrollable external factors, such as hypoxia, the absence of oxygen, whereas apoptosis is a controlled destruction of the cell. This study showed that areas of hypoxia and necrosis, but not normoxia, displayed the highest resistance to chemotherapy. Those same areas that were cut off from oxygen supply via blood flow were also cut off from chemotherapeutic drugs traveling through the circulation.

Furthermore, Zschenker et al. (2012) demonstrated that tumorospheres showed distinct expression patterns of genes responsible for extracellular matrix organization and cell adhesion. Whole-genome cDNA microarray analysis revealed significant gene expression differences indicating that cellular responsiveness to external stressors such as chemotherapeutic drugs was influenced by genes involved in integrin signaling, cell shape maintenance, and cellcell desmosome contact. All of these elements are critical to cells' association with one another in a tumorosphere.

There were two most significant correlations between tumorosphere chemoresistance and the extracellular matrix. First, the expression of vimentin, an intermediate filament found in mesenchymal, unbinded cells, was surprising because the mesenchymal phenotype typically correlates with single CTCs, which lack cell-cell adhesion. Second, the lack of expression of cortical E-cadherin, a cell adhesion molecule found in epithelial, binded cells, 
raises further questions about the cell-cell adhesion in tumorospheres (Pease et al., 2012). The epithelial-mesenchymal transition (EMT) in cancer cells causes epithelial cells to lose their polarity, cell-cell adhesion, and gain migratory and invasive properties of mesenchymal stem cells. The EMT has many transient states, with cells often displaying a mix of epithelial and mesenchymal characteristics. The EMT may be responsible for this discrepancy in vimentin and Ecadherin expression. (Figure 2) Intriguingly, subsequent research found that the chemoresistance of tumorospheres could be entirely reversed by chemically dissociating the clustered cells from one another. The changes found by Zschenker et al. mainly affected the extracellular matrix in contrast to DNA repair, which lacked significant alterations. Repairs of the genome are one factor that could lead to decreased apoptosis.

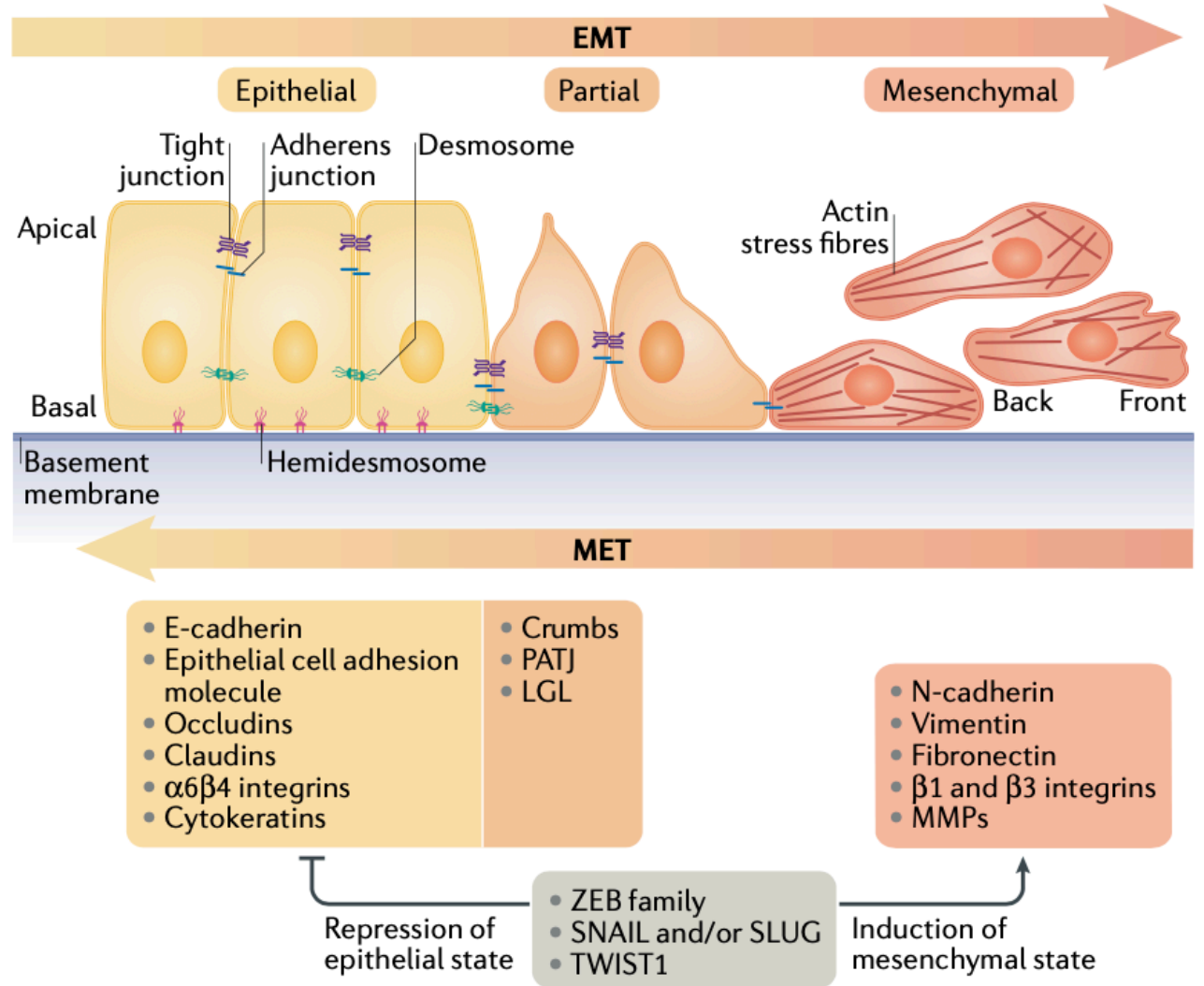

Figure 2. Outline of a typical EMT program. Induction of epithelial-mesenchymal transition (EMT) leads to the progressive loss of epithelial features and the acquisition of a partial set of mesenchymal features. During EMT, cells become motile and acquire invasive capacities. EMT is a reversible process, and mesenchymal cells can revert to the epithelial state by undergoing mesenchymal-epithelial transition (MET). It should be noted, however, that carcinoma cells in spontaneously arising tumors only very rarely advance into a completely mesenchymal state. (Figure from Dongre, A., \& Weinberg, 2019)

\section{Apoptosis}

A study done by Jansson et al. (2016) investigated the prognostic value of apoptosis, a molecular program of cell death, in single CTCs and CTC clusters for metastatic breast cancer. First, they determined that apoptosis was only observed in single CTCs; they did not find apoptotic cells in CTC clusters. They then used standard survival analysis to relate apoptosis in single CTCs and the number of CTC clusters with patient outcomes. Patients whose blood 
samples showed either more apoptosis in single CTCs or a greater quantity of CTC clusters had poorer survival, suggesting that these non-apoptotic CTC clusters alone could worsen patient survival. Clustered CTCs may have evaded anoikis, a form of programmed cell death experienced by tumor cells no longer receiving signaling from the extracellular matrix, by expressing intracellular junction proteins that maintain these survival signals. Thus, instead of mesenchymal state proteins, CTC clusters still expressed proteins associated with an epithelial state.

In 2011, Hou et al. also found that apoptosis was absent in CTC clusters while present in a subpopulation of single CTCs. This might result from CTC clusters expressing autocrine pro-migratory factors and matrix proteases needed for migration and the insulation of cells in the core of the cluster from immune attack.

Since apoptosis maintains appropriate cell numbers in healthy tissues, and since cellular interactions with the ECM affect apoptosis during cell cycle regulation, it is thought that the ECM might contribute to proper cell death. Supporting this hypothesis, Frisch et al. (1994) showed that untethered epithelial cells could evade anoikis via overexpression of BCL-2, an apoptosis inhibitor. Because CTC clusters are known to be mainly composed of epithelial cells, in contrast to single CTCs, which appear to be mesenchymal cells, this could explain why CTC clusters experience no apoptosis (Lambert et al., 2017).

In contrast to the other studies mentioned above, Jansson et al. reported higher significance for prognostic information in samples taken after several treatment cycles. Thus, early-stage CTC clusters may carry less prognostic information than initially thought, perhaps due to transient changes during the EMT.

\section{Stemness}

A third outstanding characteristic of CTC clusters is their distinct methylation patterns which resemble that of embryonic stem cells (ESCs). In 2019, Gkountela et al. investigated CTC cluster binding sites for several transcription factors (TFs) associated with stemness and proliferation. Specifically, they found that binding sites for four critical TFs - OCT4, SOX2, NANOG, and SIN3A - were hypomethylated in CTC clusters versus single CTCs. Accordingly, these stemness genes were more likely to be transcribed by RNA polymerase in CTC clusters. It should be noted that single CTCs also had hypomethylated binding sites for MEF2C, JUN, MIXL1, and SHOX2, but these TFs were not related to features of pluripotent cells.

To further test whether the clustering of CTCs directly determined methylation patterns in these four TFs, they applied FDA-approved compounds to dissociate the CTC clusters into single CTCs. Upon dissociation, they confirmed that the CTCs recovered normal methylation levels of the four critical stemness-related TFs without changing global methylation. To link these methylation changes to metastatic potential, the authors injected drug-dissociated single CTCs into mice and found that they became less likely to form metastatic lesions in vivo. Non-dissociated CTC clusters were more likely to form lesions due to hypomethylation of the four TFs.

Gkountela et al. also analyzed methylation patterns specifically within promoter and enhancer regions to determine the likelihood of transcription. This analysis revealed that CTC clusters had hypermethylation of the targets of PRC2, a repressive protein complex. PRC2 belongs to a family of Polycomb-group proteins which are essential in early metazoan development. Their role in adult humans is poorly understood (Lee et al., 2006). PRC2-mediated methylation silences the expression of its target genes, which are activated during ESC differentiation, indicating that PRC2 suppresses these genes to keep ESCs in a pluripotent state. Thus, the hypermethylation of these pro-differentiation genes by PRC2 keeps CTC clusters in a stem-like state, perhaps contributing to its metastatic potential.

Using bisulfite-sequencing data from The Cancer Genome Atlas (TCGA), Gkountela et al. found that over 30\% of the regions that were hypomethylated in CTC clusters were also hypomethylated in primary tumors of breast cancer patients. Patients with the lowest quantile of methylation levels in these regions had a significantly poorer prognosis, as measured by low progression-free survival.

Gkountela et al. confirmed their DNA methylation findings at the RNA level. They performed single-cell RNA-Seq of single CTCs and CTC clusters from liquid biopsies of breast cancer patients. They found higher counts of RNA 
from proliferation genes in CTC clusters compared to single CTCs. Two co-expression modules were significantly enriched in CTC clusters; none were significantly enriched in single CTCs. The two modules enriched in CTC clusters were related to cell-cell junctions, cellular proliferation, and platelet activation. CTC clusters use these platelets to shield themselves from NK cells and shear circulation stress, which helps them proliferate and stay clustered. Finally, to validate their RNA-Seq findings, the team stained all CTCs with Ki67 to find that CTC clusters had more Ki67high cells than single CTCs. Corresponding with their DNA methylation patterns and RNA-Seq results, CTC clusters had a higher proliferation rate, a characteristic of stem cells. Comparatively, the immunostaining results of Klameth et al. remain true; cells inside tumorospheres are quiescent (Ki67-low), even though the tumorospheres overall display Ki67-high cells.

Using the same RNA-Seq dataset, the team asked whether stemness-related genes were enriched in CTC clusters as well. They focused their analysis on 335 genes previously shown to be upregulated in ESCs by Wong et al. (2008). Gkountela et al. found that two co-expression modules containing stemness-related genes were enriched in CTC clusters; two other co-expression modules containing metabolism regulation were enriched in single CTCs.

The detailed description of epigenetic features of CTC clusters by Gkountela et al. hint at the molecular programs at work within these clusters. Much is already known about the stemness and proliferation modules they identified. Earlier studies revealed that ESCs harbor a network of TFs that prevent their differentiation while promoting proliferation; ESCs continue to self-renew because of epigenetic processes under the control of this TF network (Niwa et al., 2007). The target genes of these TFs fall under two classes: if bound by one or a few factors, the target is repressed, but if bound by more than four, the target gene is largely active (Kim et al., 2008). Interestingly, the role of one TF remains unclear; SIN3A can induce pluripotency in stem cells when paired with HDAC to form a corepressor complex (Saunders et al., 2017).

A study by Reddington et al. (2014) discussed methylation reprogramming associated with cancer which leads to alterations in the Polycomb binding landscape. Since the Polycomb network regulates cell fate and the cell cycle, any mistargeting could drive cancer formation and proliferation. Another study defined profiles of aberrant DNA methylation in renal cell carcinomas. The authors found that the expression of EZH2, a component of PRC2, associates with the overall methylation state. Because PRC2 represses differentiation genes, EZH2 should be an important target for drug developers aiming to suppress CTC cluster stemness. They further determined that Polycomb group targets were significantly enriched in tumors with the greatest methylation distinction from healthy kidney tissue (Avissar-Whiting et al., 2011). These findings outline how PRC2-mediated repression of pro-differentiation genes helps maintain the stemness of CTC clusters.

\section{Discussion}

Recent findings of chemoresistance, lack of apoptosis, and epigenetic remodeling of stemness and proliferation genes in CTC clusters versus single CTCs have painted a more complete picture of how CTC clusters stay alive longer and gain greater metastatic potential. (Figure 3) By forming clusters, CTCs can physically deter chemical infiltration, in part by maintaining a shield of ECM proteins and in part by decreasing the surface area-to-volume ratio (Klameth $e t$ al., 2017). The ratio decreases because the volume in question refers to the protoplasm, the living part of the cell, not the necrotic cores. This shield and decrease in the surface area-to-volume ratio keep CTC clusters alive during chemotherapy. CTC clusters can also escape apoptosis, which again points to the expression of ECM proteins as a potential reason for this escape (Jansson et al., 2016). This prevents CTC clusters from being directed towards regulated cell death. Within the cells of CTC clusters, subtle yet specific epigenetic events maintain the expression of important networks of stemness and proliferation genes (Gkountela et al., 2019). Stemness genes increase the capacity of these cells to become a metastatic tumor once they implant at a new site, and proliferation genes keep the cells' multiplying at a high rate. Both sets of genes make CTC clusters more formidable metastatic agents. Further research is required to determine whether these epigenetic changes occur downstream of signaling events initiated in the ECM or as an entirely separate, independent process. 


\section{Journal of Student Research}

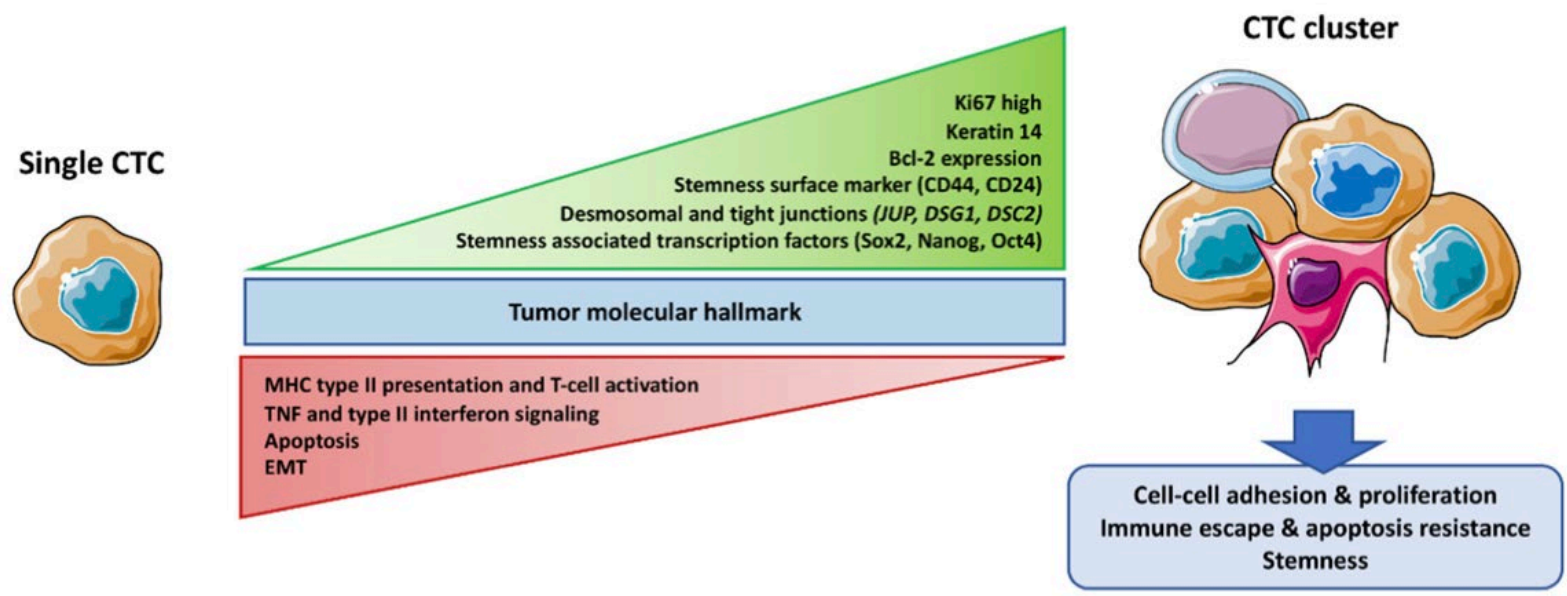

Figure 3. Molecular differences between CTC clusters and single CTCs. CTC clusters like single CTCs harbor the molecular hallmark of their primary tumor. By contrast, multiple pathways like cell-cell adhesion (desmosomes), stemness (surface markers and associated transcription factors), and proliferation (higher Ki67) are upregulated in CTC clusters. On the other hand, apoptosis and immune activation pathways are downregulated. (Figure from Amintas et al., 2020)

The interdependence of chemoresistance, apoptosis, and stemness also merits further investigation. The answer to these questions determines how many points of attack drug developers might hope to have in curbing the destructive potential of CTC clusters. Furthermore, an approximate timeline of these events could explain the progression-free survival model and help attack the metastasizing CTC clusters early on.

Finally, it is important to note the experimental limitations of these studies. According to Pampaloni et al. (2007) and Hoffman et al. (2015), the formation of tumorospheres via laboratory techniques such as low adherence cultures and 'hanging drops' has not been validated to be identical to the formation of tumorospheres in vivo. Thus, artificially prepared tumorospheres may have fundamental biological differences from CTC clusters formed in vivo. At present, there is no standard technique to study tumorospheres in vivo or in vitro. Ostensibly, the organotypic explant culture method most closely replicates a natural setting. (Figure 4)

Many independent parties have attempted to characterize CTC clusters in recent years. One of the main hurdles the field still faces is standardizing a method of study of CTC clusters in the lab. Some derive cell lines generated from CTCs of small-cell lung cancer patients, then wait for those cells to form tumorospheres in the dish (Klameth et al., 2017). While this in vitro model captured some aspects of CTC cluster biology, it is not entirely the same as CTC clusters found in organotypic explant culture in cancer patients because single CTC-derived clusters may only undergo the observed changes in vivo, not in a dish. Others force cells to cluster by gravity within a "hanging drop' or in rotating-wall vessels. Although these spheroids express a tissue-like phenotype, they can so far only form monolayer clusters. Still, others use air-medium porous membranes, artificial skin, or microscaled materials to form CTC clusters. Anchorage dependence, a prominent feature of cancer cells, remains an obstacle in these cultures. CTC clusters are often defined as groupings of two or more cancer cells, while tumorospheres grown in the lab could easily surpass that size. It remains to be seen how similar tumorospheres in vitro are to CTC clusters in vivo and whether some of the conclusions drawn from the study of tumorospheres might be unfounded. Below are a few culture models for CTC clusters. 


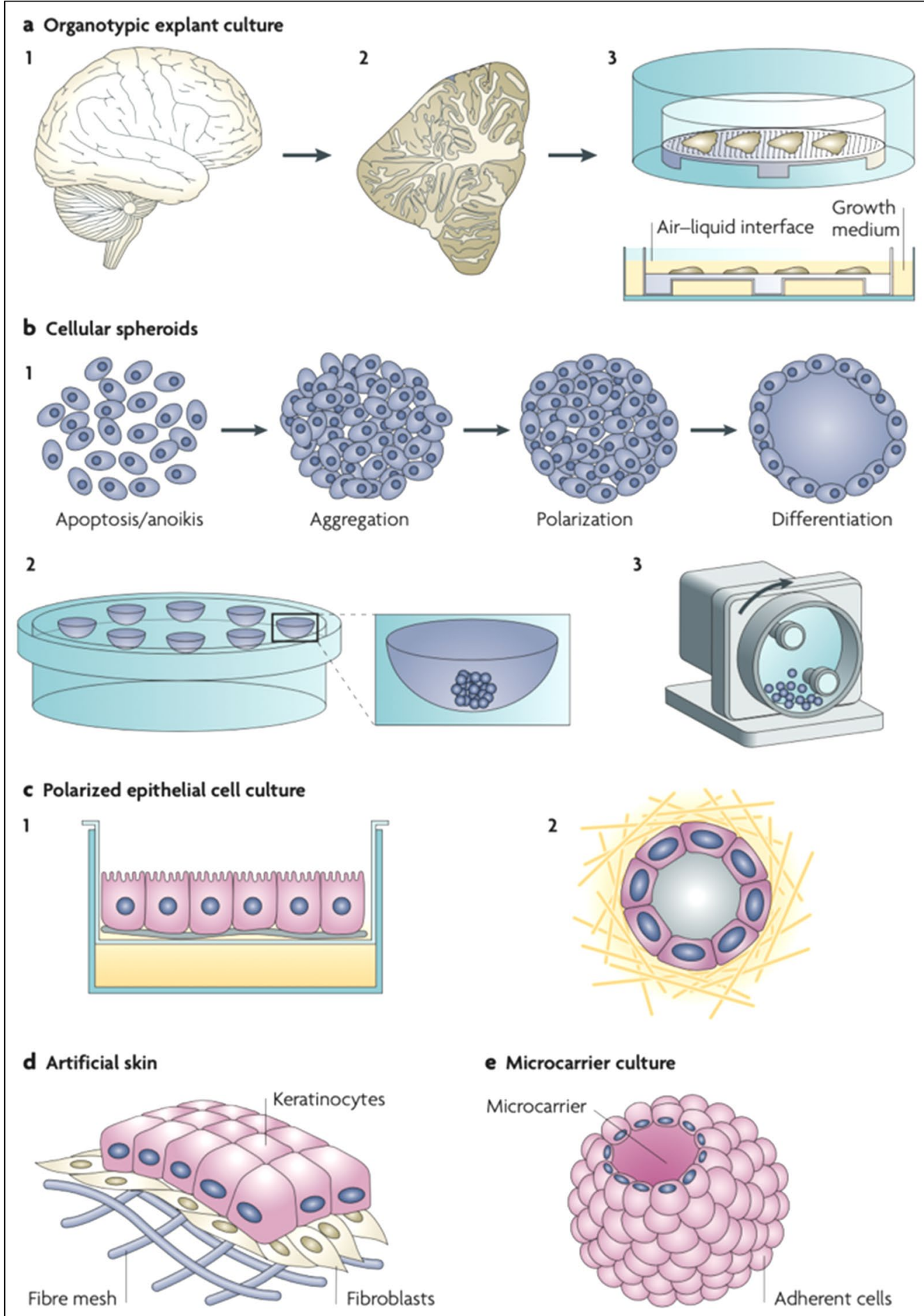

Figure 4. Three-dimensional culture models. (Figure from Pampaloni et al., 2007) 


\section{Acknowledgments}

This review would not have been possible without the extraordinary guidance and support from my advisor, Dean Lee. His experience with oncology initially encouraged me to explore the topic of CTC clusters. Dean spent many a Friday and Saturday nights rereading every version of this paper, using his incredible attention to detail to pinpoint areas of improvement. Dean's enthusiasm and care kept me on track to the final draft of this paper.

\section{References}

1. Klameth, L., Rath, B., Hochmaier, M., Moser, D., Redl, M., Mungenast, F., Gelles, K., Ulsperger, E., Zeillinger, R., \& Hamilton, G. (2017). Small cell lung cancer: model of circulating tumor cell tumorospheres in chemoresistance. Scientific reports, 7(1), 5337. https://doi.org/10.1038/s41598-017-05562-Z

2. Laurent, J., Frongia, C., Cazales, M., Mondesert, O., Ducommun, B., \& Lobjois, V. (2013). Multicellular tumor spheroid models to explore cell cycle checkpoints in 3D. BMC cancer, 13, 73. https://doi.org/10.1186/14712407-13-73

3. Hamilton, G., Rath, B., Holzer, S., \& Hochmair, M. (2016). Second-line therapy for small cell lung cancer: exploring the potential role of circulating tumor cells. Translational lung cancer research, 5(1), 71-77. https://doi.org/10.3978/j.issn.2218-6751.2015.12.12

4. Tannock, I. F., Lee, C. M., Tunggal, J. K., Cowan, D. S., \& Egorin, M. J. (2002). Limited penetration of anticancer drugs through tumor tissue: a potential cause of resistance of solid tumors to chemotherapy. Clinical cancer research : an official journal of the American Association for Cancer Research, 8(3), 878-884.

5. Däster, S., Amatruda, N., Calabrese, D., Ivanek, R., Turrini, E., Droeser, R. A., Zajac, P., Fimognari, C., Spagnoli, G. C., Iezzi, G., Mele, V., \& Muraro, M. G. (2017). Induction of hypoxia and necrosis in multicellular tumor spheroids is associated with resistance to chemotherapy treatment. Oncotarget, 8(1), 1725-1736. https://doi.org/10.18632/oncotarget.13857

6. Zschenker, O., Streichert, T., Hehlgans, S., \& Cordes, N. (2012). Genome-wide gene expression analysis in cancer cells reveals 3D growth to affect ECM and processes associated with cell adhesion but not DNA repair. PloS one, 7(4), e34279. https://doi.org/10.1371/journal.pone.0034279

7. Pease, J.C., Brewer, M., \& Tirnauer, J. (2012). Spontaneous spheroid budding from monolayers: a potential contribution to ovarian cancer dissemination. Biology Open, 1, 622 - 628. https://doi.org/10.1242/bio.2012653

8. Pampaloni, F., Reynaud, E. G., \& Stelzer, E. H. (2007). The third dimension bridges the gap between cell culture and live tissue. Nature reviews. Molecular cell biology, 8(10), 839-845. https://doi.org/10.1038/nrm2236

9. Hoffmann, O. I., Ilmberger, C., Magosch, S., Joka, M., Jauch, K. W., \& Mayer, B. (2015). Impact of the spheroid model complexity on drug response. Journal of biotechnology, 205, 14-23. https://doi.org/10.1016/j.jbiotec.2015.02.029

10. Jansson, S., Bendahl, P. O., Larsson, A. M., Aaltonen, K. E., \& Rydén, L. (2016). Prognostic impact of circulating tumor cell apoptosis and clusters in serial blood samples from patients with metastatic breast cancer in a prospective observational cohort. BMC cancer, 16, 433. https://doi.org/10.1186/s12885-016-2406-y

11. Hou, J. M., Krebs, M., Ward, T., Sloane, R., Priest, L., Hughes, A., Clack, G., Ranson, M., Blackhall, F., \& Dive, C. (2011). Circulating tumor cells as a window on metastasis biology in lung cancer. The American journal of pathology, 178(3), 989-996. https://doi.org/10.1016/j.ajpath.2010.12.003

12. Frisch, S. M., \& Francis, H. (1994). Disruption of epithelial cell-matrix interactions induces apoptosis. The Journal of cell biology, 124(4), 619-626. https://doi.org/10.1083/jcb.124.4.619

13. Lambert, A. W., Pattabiraman, D. R., \& Weinberg, R. A. (2017). Emerging Biological Principles of Metastasis. Cell, 168(4), 670-691. https://doi.org/10.1016/j.cell.2016.11.037 
14. Gkountela, S., Castro-Giner, F., Szczerba, B. M., Vetter, M., Landin, J., Scherrer, R., Krol, I., Scheidmann, M. C., Beisel, C., Stirnimann, C. U., Kurzeder, C., Heinzelmann-Schwarz, V., Rochlitz, C., Weber, W. P., \& Aceto, N. (2019). Circulating Tumor Cell Clustering Shapes DNA Methylation to Enable Metastasis Seeding. Cell, 176(1-2), 98-112.e14. https://doi.org/10.1016/j.cell.2018.11.046

15. Lee, T. I., Jenner, R. G., Boyer, L. A., Guenther, M. G., Levine, S. S., Kumar, R. M., Chevalier, B., Johnstone, S. E., Cole, M. F., Isono, K., Koseki, H., Fuchikami, T., Abe, K., Murray, H. L., Zucker, J. P., Yuan, B., Bell, G. W., Herbolsheimer, E., Hannett, N. M., Sun, K., ... Young, R. A. (2006). Control of developmental regulators by Polycomb in human embryonic stem cells. Cell, 125(2), 301-313. https://doi.org/10.1016/j.cell.2006.02.043

16. Wong, D. J., Liu, H., Ridky, T. W., Cassarino, D., Segal, E., \& Chang, H. Y. (2008). Module map of stem cell genes guides creation of epithelial cancer stem cells. Cell stem cell, 2(4), 333-344. https://doi.org/10.1016/j.stem.2008.02.009

17. Niwa H. (2007). How is pluripotency determined and maintained?. Development (Cambridge, England), 134(4), 635-646. https://doi.org/10.1242/dev.02787

18. Kim, J., Chu, J., Shen, X., Wang, J., \& Orkin, S. H. (2008). An extended transcriptional network for pluripotency of embryonic stem cells. Cell, 132(6), 1049-1061. https://doi.org/10.1016/j.cell.2008.02.039

19. Saunders, A., Huang, X., Fidalgo, M., Reimer, M. H., Jr, Faiola, F., Ding, J., Sánchez-Priego, C., Guallar, D., Sáenz, C., Li, D., \& Wang, J. (2017). The SIN3A/HDAC Corepressor Complex Functionally Cooperates with NANOG to Promote Pluripotency. Cell reports, 18(7), 1713-1726. https://doi.org/10.1016/j.celrep.2017.01.055

20. Reddington, J. P., Sproul, D., \& Meehan, R. R. (2014). DNA methylation reprogramming in cancer: does it act by re-configuring the binding landscape of Polycomb repressive complexes?. BioEssays : news and reviews in molecular, cellular and developmental biology, 36(2), 134-140. https://doi.org/10.1002/bies.201300130

21. Avissar-Whiting, M., Koestler, D. C., Houseman, E. A., Christensen, B. C., Kelsey, K. T., \& Marsit, C. J. (2011). Polycomb group genes are targets of aberrant DNA methylation in renal cell carcinoma. Epigenetics, 6(6), 703-709. https://doi.org/10.4161/epi.6.6.16158

22. Dongre, A., \& Weinberg, R. A. (2019). New insights into the mechanisms of epithelial-mesenchymal transition and implications for cancer. Nature reviews. Molecular cell biology, 20(2), 69-84.

https://doi.org/10.1038/s41580-018-0080-4

23. Amintas, S., Bedel, A., Moreau-Gaudry, F., Boutin, J., Buscail, L., Merlio, J. P., Vendrely, V., Dabernat, S., \& Buscail, E. (2020). Circulating Tumor Cell Clusters: United We Stand Divided We Fall. International journal of molecular sciences, 21(7), 2653. https://doi.org/10.3390/ijms21072653 